\title{
Assessment of effect of nasal continuous positive pressure on laryngeal opening using fibre optic laryngoscopy
}

P Gaon, S Lee, S Hannan, D Ingram, A D Milner

\begin{abstract}
Aim-To assess the effect of nasal continuous positive airways pressure (CPAP) on the dimensions of the laryngeal opening. Methods-Nine preterm infants who had previously received ventilatory support for respiratory distress syndrome (RDS) were studied. All were receiving nasal CPAP. The laryngeal opening was visualised using a fibre optic video camera system. The ratio of width to length of the opening was measured on and off CPAP.

Results-In eight of the infants the width: length ratio increased on CPAP; mean change for group $+24.4 \%(95 \% \mathrm{CI}+11.9$ to +37.9 ).

Conclusions-Nasal CPAP seems to dilate the larynx. This may explain the selective beneficial effects of CPAP on mixed and obstructive apnoea.
\end{abstract}

(Arch Dis Child Fetal Neonatal Ed 1999;80:F230-F232)

Keywords: nasal continuous positive airway pressure; apnoea of prematurity; laryngoscopy; vocal cords

The use of continuous positive airway pressure (CPAP) for the treatment of apnoea of prematurity was first described in $1975 .{ }^{1}$ Since then studies have shown that CPAP roughly halves the incidence of apnoeic episodes. ${ }^{2}$ Several mechanisms have been suggested to explain this effect. CPAP may stabilise the chest wall during breathing which could eliminate the suppressant effect of the intercostal-phrenic inhibitory reflex, stimulated by chest wall distortion in preterm infants. ${ }^{3}$ An alternative explanation is that the improvement in oxygenation which often accompanies the use of CPAP is responsible. ${ }^{4}$ Other studies have suggested that stimulation of the Hering Breuer inflation reflex may be responsible. ${ }^{5}$ It is now well established that the apnoea of prematurity can be divided into three types - namely central, obstructive, and mixed. ${ }^{67}$ Miller and colleagues $^{8}$ showed that CPAP was highly effective as a treatment for mixed and obstructive apnoea, but that it did not reduce the incidence of central apnoea. This raised the possibility that airway closure may be the primary event in mixed apnoea, acting via the intercostal-phrenic inhibitory reflex so that CPAP prevents airway closure rather than by directly affecting central respiratory drive.

The two most probable sites for upper airway obstruction are in the pharynx and at the level of the larynx. The pharynx is composed entirely of soft tissues and kept patent during inspiration by the opposing actions of the genioglossal and geniohyoid muscles. ${ }^{9}$ Any reduction in the tone of these muscles will tend to lead to obstruction. This has been well documented as the cause and site of obstruction in children and adults presenting with obstructive sleep apnoea. ${ }^{10} 11$ It is also well established that CPAP is a useful form of treatment in both adults and children with this problem. ${ }^{12} 13$

The larynx is the narrowest part of the neonatal airway. Although Mathew and colleagues ${ }^{14}$ documented high pharyngeal closure during mixed and obstructive apnoea in preterm infants, using pharyngeal catheters, Ruggins et $a l^{15}$ showed that the entrance to the larynx was obstructed in preterm infants with problematic apnoea, due to adduction of the arytenoid masses and the aryepiglottic folds. These studies both suggest, therefore, that there is obstruction at both the laryngeal entrance and as a secondary phenomenon, in the pharynx. Whatever the site, CPAP may prevent apnoea by distending the upper airway, thus reducing airway closure. This study therefore aimed to assess the effects of $5 \mathrm{~cm} \mathrm{H}_{2} \mathrm{O}$ of CPAP on the dimensions of the laryngeal opening.

\section{Methods}

A $2.2 \mathrm{~mm}$ fibre optic angioscope (Olympus PF-22) was used to visualise the laryngeal opening. The tip of the scope is flexible through about 120 degrees in one plane. This movement is controlled by a hand piece. The endoscope has a light source and is attached to a wide angle camera (OTV-S2 Olympus) which in turn is connected to a colour monitor so that images can be viewed continuously. The images from the screen were also recorded on a videotape recorder.

The technique required the presence of two investigators, one to pass and control the endoscope, the other to maintain the position of the baby, check the monitoring systems, provide suction, and record the readings of the video counter whenever CPAP was started or discontinued. A CPAP pressure of $5 \mathrm{~cm} \mathrm{H}_{2} \mathrm{O}$ was used for all babies. Heart rate and transcutaneous saturation were recorded continuously.

If the babies were receiving oral feeds, their stomachs were aspirated to reduce the risk of aspiration. Oro-phayngeal suction was also carried out after the babies had been positioned supine, with mild neck extension using a neck support. While continuing to receive CPAP, the endoscope tip was passed through 

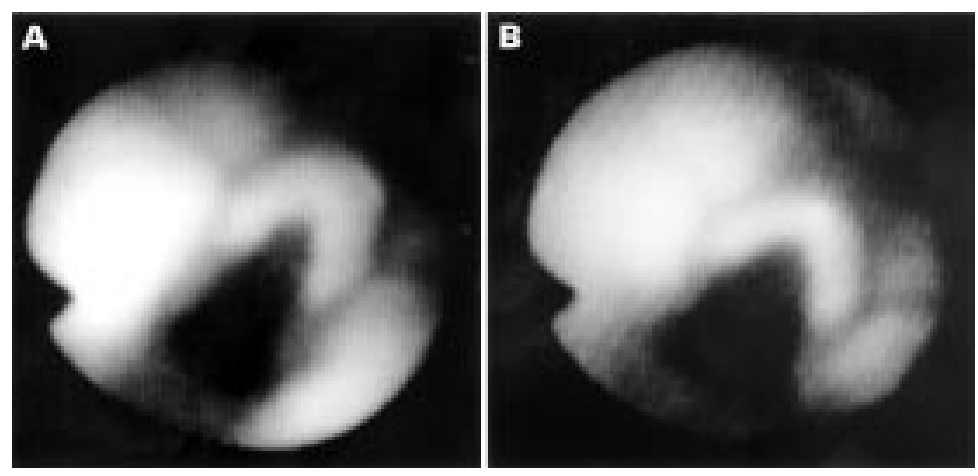

Figure 1 Photographs of maximal laryngeal opening: $(A)$ off and (B) on CPAP in the same infant.
Table 1 Width:length ratios of the laryngeal opening, off and on CPAP (mean and coefficient of variation) and percentage change in ratio in nine infants studied

\begin{tabular}{llll}
\hline Case No & Off CPAP & On CPAP & \% Change \\
\hline 1 & $0.47(14.9)$ & $0.69(21.7)$ & +47 \\
2 & $0.73(17.8)$ & $0.88(21)$ & +21 \\
3 & $0.64(6.9)$ & $0.72(9.9)$ & +12.5 \\
4 & $0.58(6.9)$ & $0.71(9.9)$ & +22.5 \\
5 & $0.43(25.6)$ & $0.63(9.5)$ & +46.5 \\
6 & $0.56(10.7)$ & $0.74(21.6)$ & +32.1 \\
7 & $0.76(0)$ & $0.92(31.5)$ & +21 \\
8 & $0.6(3.3)$ & $0.75(13.3)$ & +25 \\
9 & $0.68(10.3)$ & $0.64(14.1)$ & -6 \\
Mean & $0.61(11.8)$ & $0.74(15.6)$ & +24.6 \\
\hline
\end{tabular}

\section{Results}

Initially the introduction of the endoscope stimulated a gag reflex but this was short lived and the babies tolerated the presence of the endoscope well. The picture obtained of the larynx was intermittently lost as a result of swallowing, the build up of secretions, or changes in posture of the baby. The mean number of breaths analysed in each baby was 6.1 on CPAP and 5.1 off CPAP.

Eight of the nine babies studied had a mean width:length ratio greater on than when off CPAP. (table 1). The mean difference in the ratio produced by CPAP for the total group was $+24 \%$ with $95 \%$ confidence intervals of +12 to $+37.1 \%$.

\section{Discussion}

Our results show that CPAP increases the laryngeal opening of preterm infants during inspiration. Direct measurements on pharyngeal structures cannot be made, using this technique, as there are no identifiable markers, and no means for directly calibrating the size of objects or spaces. We therefore used the ratio of the width to the length of the laryngeal opening during maximum abduction of the vocal cords. This consistently occurred during inspiration. Of the eight babies who showed this change in response to CPAP, the ratio of width to length increased by 12.5 to $47 \%$. The remaining baby had a reduction of $6 \%$. This represented less than half the mean coefficient of variation for the measurements off CPAP. We are unable to state the extent to which these changes are pressure sensitive as measurements were only obtained at a single pressure of $5 \mathrm{~cm} \mathrm{H}_{2} \mathrm{O}$.

Although CPAP could have produced these changes by stimulating greater abduction of the vocal cords, the most likely explanation for the changes observed is that CPAP has a distending effect on the upper airway, holding open the relatively compliant larynx of the preterm baby. Studies on adults show that this distending pressure would also prevent pharyngeal collapse, although for the reasons stated above, we were unable to confirm this due to the limitations of our technique. The implications of this study are that CPAP will tend to prevent spontaneous upper airway closure in preterm babies. We have evidence from our previous study ${ }^{11}$ that upper airway closure occurs at the entrance to the larynx both in mixed as well as obstructive apnoea. We postulate that this airway closure is an 
important aetiological factor in mixed apnoea, leading to significant deformation of the chest wall as the baby makes respiratory efforts against the obstructed airway. This will stimulate the central apnoea component of mixed apnoea via the intercostal-phrenic inhibitory reflex, a respiratory suppressant reflex produced by chest wall deformity. ${ }^{3}$ This is fully consistent with the findings of Miller and colleagues ${ }^{8}$ and goes some way to explaining their observation that CPAP only reduces the incidence of mixed and obstructive apnoea.

Our study could be criticised on the grounds that we did not select preterm babies who had troublesome apnoea, in whom it might have been possible to show a direct link between the observed changes and the prevention of apnoeic attacks. This would have been a more difficult and time consuming task, and we have no reason to consider that the changes would have been different. A further possible criticism is that all these babies had recently received intermittent positive pressure ventilation via an endotracheal tube which could have persisting effects on laryngeal function. This again we consider unlikely as all the babies had been extubated for over 24 hours.

In conclusion, we have shown that nasal CPAP tends to increase the width of the laryngeal opening, and consider that this provides an explanation for the selective preventive effect of CPAP on mixed and obstructive apnoea in preterm babies.
We thank the Tommy's Campaign who generously funded S Lee, the Foundation for the Study of Infant Death who funded $S$ Hannam, and provided the funds for the equipment, and The Special Trustees of St Thomas's Hospital who funded D Ingram.

1 Kattwinkel J, Nearman HS, Fanaroff AA, Katona PG, Klau MH. Apnoea of prematurity. F Pediatr 1975; 86:588-92.

2 Spiedel BD, Dunn PM. Use of nasal continuous positive airway pressure to treat severe recurrent apnoea in very preterm infants. Lancet: 1976;ii:658-60.

3 Hagan R, Bryan AC, Bryan MH, Gulston G. Neonatal chest wall afferents and regulation of respiration. $\mathcal{F}$ Appl Physiol 1977;42:362-7.

4 Durand M, McCann E, Brady JP. Effect of continuous positive airways pressure on the ventilatory response to $\mathrm{CO}_{2}$ in in preterm infants. Pediatrics 1983;71:634-8.

5 Martin RJ, Nearman HS, Katona PG, Klaus MH. The effect of a low continuous positive airway pressure on the reflex control of respiration in the preterm infant. $\mathcal{f}$ Pediatr 1977;90: $976-81$.

6 Dransfield DA, Fox WW. A non-invasive method for recording central and obstructive apnea with bradycardia in infants. Crit Care Med 1980;8:663-6.

7 Upton CJ, Milner AD Stokes GM. Upper airway patency during apnoea of prematurity. Arch Dis Child 1992; 67:419-24.

8 Miller MJ, Carlo WA, Martin RJ. Continuous positive airways pressure selectively reduces obstructive apnoea in preterm infants. F Pediatr 1985; 106:91-4

9 Brouillette RT, Thach BT. A neuromuscular mechanism maintaining extrathoracic airway patency. I Appl Physiol 1979;46:772-9.

10 Guillerminault G, Hill WH, Simmons FB, Dement WC. Obstructive sleep apnoea: electromyographic and fibreObstructive sleep apnoea: electromyograph
optic studies. Exp Neurol 1978; 62: 48-67.

11 Southall DP, Croft CB, Stebbens VA. Detection of sleep associated dysfunctional pharyngeal obstruction in inassociated dysfunctional pharynge

12 Sullivan CE, Issa FG, Berthen-Jones M, Evesa L. Reversal of obstructive sleep apnea by continuous positive airway pressure applied through the nares. Lancet 1981;i:862-5.

13 Marcus CL, Ward SL, Mallory GB, et al. Use of continuous positive airway pressure as a treatment of childhood obstructive sleep apnea. $\mathcal{F}$ Pediatr 1995;127:88-94.

14 Mathew OP, Roberts JL, Thach BT. Pharyngeal airway obstruction in preterm infants during mixed and obstructive apnoea. F Pediatr 1982;100:964-8.

15 Ruggins NR, Milner AD. Site of upper airway obstruction in preterm infants with problematic apnoea. Arch Dis Child 1991;66:787-92. 